\title{
A Practical Predictive Model Based on Ultrasound Imaging and Clinical Indices for Estimation of Response to Neoadjuvant Chemotherapy in Patients with Breast Cancer
}

\section{Pingping $\mathrm{Ye}^{\mathrm{I}}$ \\ Hongbo Duan' \\ Zhenya Zhao ${ }^{2}$ \\ Shibo Fang $\mathbb{D}^{\prime}$}

'Department of Ultrasonography, The Sixth Hospital of Ningbo City of Zhejiang Province, Ningbo, 315100, People's Republic of China; ${ }^{2}$ Department of Imaging, The First Hospital of Ningbo City of Zhejiang Province, Ningbo, 315010, People's Republic of China
Correspondence: Shibo Fang Department of Ultrasonography, The Sixth Hospital of Ningbo City of Zhejiang Province, 1059 Zhongshan East Road, Ningbo, Zhejiang Province, People's

Republic of China

Tel +86 0574-87996II5

Email yepingping32I@I63.com
Purpose: Clinical responses of neoadjuvant chemotherapy (NACT) are associated with prognosis in patients with breast cancer. The selection of suitable variables for the prediction of clinical responses remains controversial. Herein, we developed a predictive model based on ultrasound imaging and clinical indices to identify patients most likely to benefit from NACT.

Patients and Methods: We recruited a total of 225 consecutive patients who underwent NACT followed by surgery and axillary lymph node dissection at the Sixth Hospital of Ning Bo City of Zhe Jiang Province between January 1, 2018, and March 31, 2021. All patients had been diagnosed with breast cancer following the clinical examination. First, we created a training cohort of patients who underwent NACT+surgery $(\mathrm{N}=180)$ to develop a nomogram. We then validated the performance of the nomogram in a validation cohort of patients who underwent NACT + surgery $(\mathrm{N}=45)$. Multivariate logistic regression was then used to identify independent risk factors that were associated with the response to NACT; these were then incorporated into the nomogram.

Results: Multivariate logistic regression analysis identified several significant differences as to clinical responses of NACT, including neutrophil-lymphocyte ratio (NLR), body mass index (BMI), pulsatility index (PI), resistance index (RI), blood flow, Ki67, histological type, molecular subtyping, and tumor size. The performance of the nomogram score exhibited a robust $\mathrm{C}$-index of 0.89 (95\% confidence interval [CI]: 0.83 to 0.95$)$ in the training cohort and a high C-index of 0.87 (95\% CI: 0.81 to 0.93$)$ in the validation cohort. Clinical impact curves showed that the nomogram had a good predictive ability.

Conclusion: We successfully established an accurate and optimized nomogram incorporated ultrasound imaging and clinical indices that could be used preoperatively to predict clinical responses of NACT. This model can be used to evaluate the risk of clinical responses to NACT and therefore facilitate the choice of personalized therapy.

Keywords: breast cancer, NACT, clinical response, nomogram prediction

\section{Introduction}

Globally, breast cancer is the most common malignant tumor among women and the second leading cause of cancer-related death every year. ${ }^{1}$ The NACT is widely offered to breast cancer patients and is mainly used to reduce tumor burden and enable breast-conserving surgery. ${ }^{2,3}$ In the past decade, NACT has provided the opportunity to assess the response to treatment by an in vivo chemosensitivity test. ${ }^{4}$ 
Indeed, several studies have demonstrated that breast cancer patients who achieve clinical responses after NACT exhibit improved prognosis. ${ }^{5-8}$ Nevertheless, only $30-73 \%$ of patients achieved clinical responses of NACT, ${ }^{9}$ meaning that the majority of patients do not benefit from NACT. Given these concerns, changes in the approach and patient selection that result in greater sensitivity have been recommended as necessary to support further treatment. Therefore, the development of a practical tool to predict the pathological response in patients with breast cancer after NACT is necessary.

Multiple models have been published predicting axillary pathological response after NACT in varying cohorts. $^{10-23}$ Some baseline clinicopathological features and genotyping can predict the curative effect of NACT for breast cancer and further reflect the satisfied prognosis. However, the high costs associated with these genetic and molecular tests limit their utility in regular clinical practice. ${ }^{21}$ The economical and practical variables selected to establish the optimal prediction model could help select the patients who will benefit from NACT and recommend a tailored approach when choosing the initial treatment. Therefore, it is vital that more readily accessible pathologic, angiographic indices, or laboratory biomarkers associated with NACT treatment outcomes be identified.

In the present study, we established a nomogram that incorporated ultrasound imaging and clinical indices to predict pathological response to NACT. We then analyzed the predictive performance of this nomogram in a deviation cohort and then verified the performance in an internal validation cohort.

\section{Patients and Methods}

\section{Patients Enrollment}

Between January 1, 2018, and March 31, 2021, we prospectively collated data from consecutive patients who had been diagnosed with breast cancer at the Sixth Hospital of Ning Bo City of Zhe Jiang Province. All patients had received NACT before surgery. This study was approved by the Institutional Ethics Committee of the Sixth Hospital of Ning Bo City of Zhe Jiang Province, in compliance with the Declaration of Helsinki. Written informed consent was obtained from all participants before any treatment. All patients' information was anonymous. The inclusion criteria were as follows: (1) All patients were diagnosed with invasive breast cancer using hollow needle biopsy before chemotherapy; (2) patient had received preoperative ultrasound imaging and routine pelvic examination, and (3) patient had undergone 2 to 6 cycles of NACT before surgery. We excluded: (1) patients who had a history of all other malignancies and any patient who had an incomplete set of medical data and (2) other types of neoadjuvant therapy, including radiotherapy and endocrine therapy. The flow chart of patient selection and data process is shown in Figure 1.

\section{Evaluating the Safety and Efficacy of NACT}

According to RECIST (version 1.1) criteria, the response to NACT is divided into four levels: (1) complete response (CR) in which the tumor completely disappeared; (2) partial response (PR) in which the diameter of the tumor was increased by at least $30 \%$; (3) progressive disease (PD) in which the diameter of the tumor was increased by at least $20 \%$; and (4) stable disease (SD): in which the diameter of the tumor did not shrink sufficiently to qualify for PR but did not increase sufficiently to qualify for PD. ${ }^{24}$

\section{Statistical Analysis}

Continuous variables are expressed as mean (standard deviation) and compared using the two-tailed $t$-test or the MannWhitney test. Categorical variables were compared using the $\chi 2$ test or Fisher's exact test. Univariate and multivariate logistic analyses were used to explore the risk factors for a pathological response. A nomogram was formulated based on results arising from the multivariate logistic regression analysis. The nomogram was based on the proportional conversion of each regression coefficient in the multivariate logistic regression to a 0 to 100 -point scale. The effect of the variable with the highest $\beta$ coefficient (the absolute value) was assigned 100 points. Points were added for all independent variables to create a total, which was then converted to predicted probabilities. Next, we used bootstrapping plots to calculate the concordance index (C-index) and area under the time-dependent receiver operating characteristic curve (timedependent AUC) so that we could evaluate our ability to calibrate the curve. Typically, C-index and AUC values that exceeded 0.6 were suggestive of reasonable estimation. The cut-off point for risk stratifications was selected using X-tile. All analysis was performed using the Python programming language (version 3.9.2, Python Software Foundation, https://www.python.org/) and R Project for Statistical Computing (version 4.0.4, http://www.r-project.org/). All $\mathrm{P}$ values were two-tailed, and $\mathrm{P}<0.05$ was considered statistically significant. 


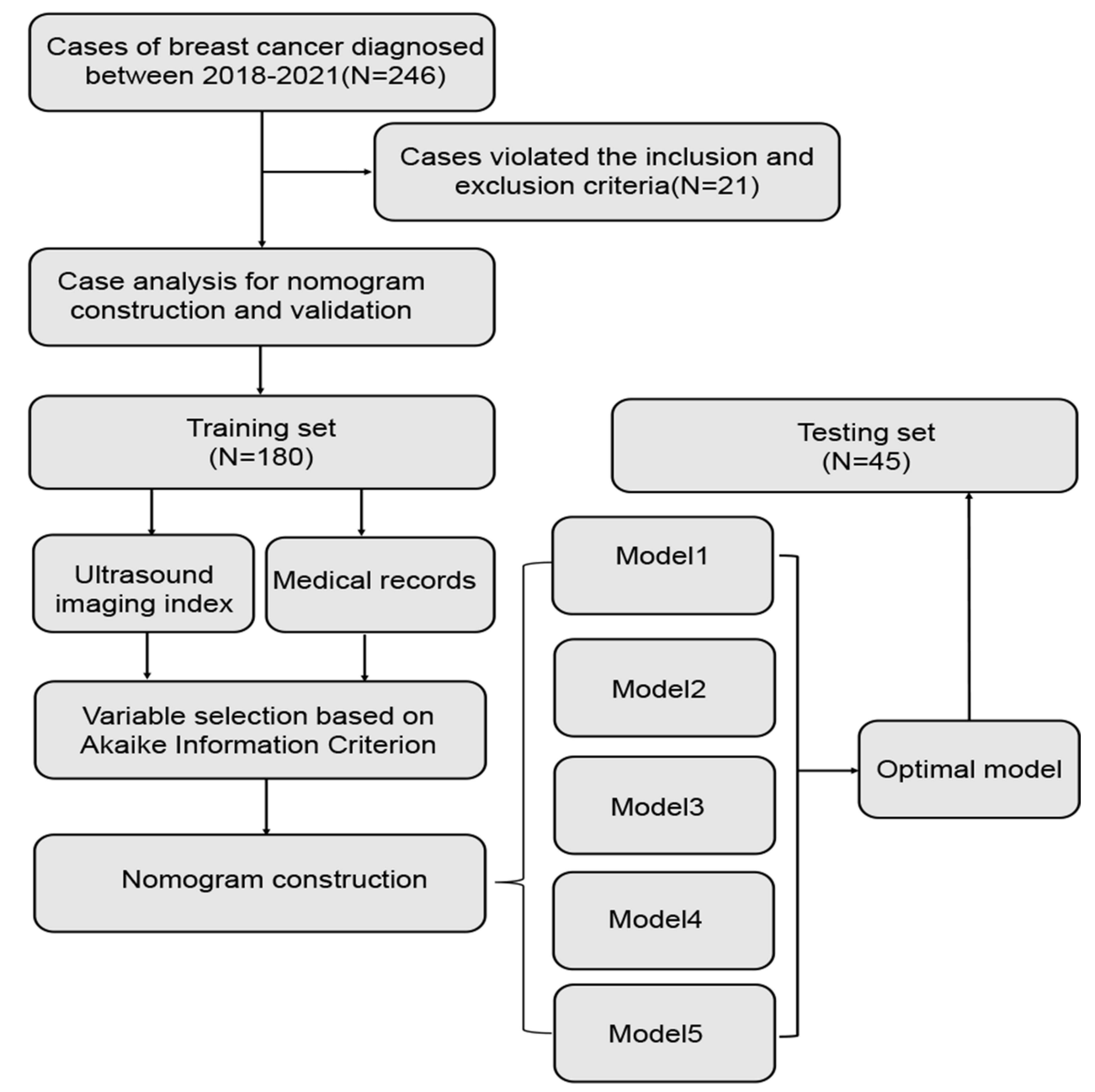

Figure I The flow chart of patient selection and data process.

\section{Results}

\section{Clinicopathological Characteristics}

During the period of enrollment, 246 consecutive patients with breast cancer underwent NACT in Sixth Hospital of Ning Bo City of Zhe Jiang Province. Of these, 180 patients met our inclusion criteria and were enrolled in our training cohort. Next, we enrolled in our validation cohort; these patients underwent procedures between May 1, 2020, and April 1, $2021(\mathrm{n}=45)$. Demographics and baseline data are summarized in Table 1. According to the RECIST (version 1.1) criteria, the clinical response was identified in 141 $(78.3 \%)$ and $21(82.2 \%)$ patients in the training and validation cohorts, respectively.

\section{Feature Selection and Model Construction} Information criteria based on penalized likelihood, such as Akaike's information criterion (AIC), are widely used for model selection in health and biological research. ${ }^{25}$ All the parameters used to construct the nomogram were based on preoperative data, including peripheral blood inflammatory markers (eg, NLR), imaging indicators (eg, the largest tumor diameter, PI, RI, blood flow), and clinicopathological parameters (eg, BMI, histological type, molecular subtyping, Ki67, BMI). In the univariable analyses, variables with $\mathrm{P}<0.05$ were included in the multivariate logistic regression analysis. The results arising from the multivariate logistic analysis are summarized in Table 2 . Based on the principle of AIC, we selected the top five AIC index models and summarized the intersection variables in the five models, including PI, RI, and blood flow (Figure 2A). Besides, the relative importance rank of all eight variables for estimation of pathological response and the five contributive models are illustrated in Figure 2B. The topweighted features (BMI, NLR, tumor size, histological type, molecular subtyping, PI, RI, and blood flow classification) were highly correlated with poor pathological 
Table I The Predictive Performances of Different Models Associated with Pathological Remission

\begin{tabular}{|c|c|c|c|c|c|c|c|c|}
\hline \multirow[t]{2}{*}{ Model } & \multicolumn{4}{|c|}{$95 \% \mathrm{Cl}$} & \multicolumn{4}{|c|}{ Discrimination } \\
\hline & OR & Lower & Upper & P-value & Brier & $\mathbf{R}^{2}$ & C-Index & AIC \\
\hline ModelI & & & & & 0.04 & 0.06 & 0.882 & 325.70 \\
\hline Tumor_size*, cm & 0.83 & 0.72 & 0.97 & 0.09 & & & & \\
\hline $\begin{array}{l}\mathrm{PI} \\
<0.58: \geq 0.58\end{array}$ & 1.05 & 0.87 & 1.27 & 0.02 & & & & \\
\hline $\begin{array}{l}\text { RI } \\
\geq 0.47:<0.47\end{array}$ & 1.19 & 0.93 & 1.51 & 0.04 & & & & \\
\hline $\begin{array}{l}\text { Pathology } \\
\text { IDC:SC } \\
\text { ILC:SC } \\
\text { MCB:SC }\end{array}$ & $\begin{array}{l}0.30 \\
0.48 \\
1.52\end{array}$ & $\begin{array}{l}0.14 \\
0.35 \\
0.93\end{array}$ & $\begin{array}{l}0.63 \\
0.66 \\
2.49\end{array}$ & $\begin{array}{c}0.01 \\
0.02 \\
<0.01\end{array}$ & & & & \\
\hline $\begin{array}{l}\text { Molecular_subtyping } \\
\text { Luminal:TNBC } \\
\text { HER2(+):TNBC } \\
\text { LH:TNBC }\end{array}$ & $\begin{array}{l}0.95 \\
0.58 \\
0.74\end{array}$ & $\begin{array}{l}0.74 \\
0.45 \\
0.55\end{array}$ & $\begin{array}{l}1.21 \\
0.75 \\
0.99\end{array}$ & $\begin{array}{l}<0.01 \\
0.03 \\
0.03\end{array}$ & & & & \\
\hline $\begin{array}{l}\text { Blood_flow } \\
\geq 1.88:<1.88\end{array}$ & 1.34 & 1.02 & 1.78 & 0.04 & & & & \\
\hline $\begin{array}{l}\text { NLR } \\
\geq 3.14:<3.14\end{array}$ & 1.18 & 0.67 & 2.09 & $<0.01$ & & & & \\
\hline $\begin{array}{l}\text { BMI } \\
<18: 18-24 \\
\geq 25: 18-24\end{array}$ & $\begin{array}{l}0.98 \\
1.53\end{array}$ & $\begin{array}{l}0.72 \\
1.18\end{array}$ & $\begin{array}{l}1.35 \\
1.98\end{array}$ & $\begin{array}{l}<0.01 \\
0.02\end{array}$ & & & & \\
\hline Model2 & & & & & 0.05 & 0.06 & 0.857 & 331.50 \\
\hline Tumor_size*, cm & 0.78 & 0.39 & 1.17 & 0.02 & & & & \\
\hline $\begin{array}{l}\mathrm{PI} \\
<0.58: \geq 0.58\end{array}$ & 1.23 & 0.84 & 1.62 & 0.01 & & & & \\
\hline $\begin{array}{l}\text { Ki67 } \\
\geq 0.50:<0.50\end{array}$ & 1.14 & 0.75 & 1.53 & 0.04 & & & & \\
\hline $\begin{array}{l}\text { Pathology } \\
\text { IDC:SC } \\
\text { ILC:SC } \\
\text { MCB:SC }\end{array}$ & $\begin{array}{l}0.48 \\
0.52 \\
1.39\end{array}$ & $\begin{array}{l}0.03 \\
0.11 \\
0.94\end{array}$ & $\begin{array}{l}0.93 \\
0.93 \\
1.84\end{array}$ & $\begin{array}{c}0.04 \\
<0.01 \\
<0.01\end{array}$ & & & & \\
\hline $\begin{array}{l}\text { Molecular_subtyping } \\
\text { Luminal:TNBC } \\
\text { HER2(+):TNBC } \\
\text { LH:TNBC }\end{array}$ & $\begin{array}{l}0.47 \\
0.51 \\
0.38\end{array}$ & $\begin{array}{l}0.06 \\
0.10 \\
0.09\end{array}$ & $\begin{array}{l}0.88 \\
0.92 \\
0.67\end{array}$ & $\begin{array}{l}<0.01 \\
0.02 \\
0.04\end{array}$ & & & & \\
\hline $\begin{array}{l}\text { Blood_flow } \\
\geq 1.88:<1.88\end{array}$ & 1.25 & 0.80 & 1.71 & 0.01 & & & & \\
\hline $\begin{array}{l}\text { NLR } \\
\geq 3.14:<3.14\end{array}$ & 1.13 & 0.67 & 1.53 & $<0.01$ & & & & \\
\hline
\end{tabular}

(Continued) 
Table I (Continued).

\begin{tabular}{|c|c|c|c|c|c|c|c|c|}
\hline \multirow[t]{2}{*}{ Model } & \multicolumn{4}{|c|}{$95 \% \mathrm{Cl}$} & \multicolumn{4}{|c|}{ Discrimination } \\
\hline & OR & Lower & Upper & P-value & Brier & $\mathbf{R}^{2}$ & C-Index & AIC \\
\hline BMI & & & & & & & & \\
\hline$<18: 18-24$ & 0.89 & 0.46 & 1.32 & 0.06 & & & & \\
\hline$\geq 25: 18-24$ & 1.27 & 0.86 & 1.68 & 0.03 & & & & \\
\hline Model3 & & & & & 0.04 & 0.06 & 0.764 & 334.60 \\
\hline Age*, year & 0.67 & 0.16 & 1.18 & 0.05 & & & & \\
\hline PI & & & & & & & & \\
\hline$<0.58: \geq 0.58$ & 0.85 & 0.42 & 1.28 & 0.02 & & & & \\
\hline RI & & & & & & & & \\
\hline$\geq 0.47:<0.47$ & 1.22 & 0.81 & 1.63 & 0.04 & & & & \\
\hline Pathology & & & & & & & & \\
\hline IDC:SC & 0.38 & 0.32 & 0.79 & 0.01 & & & & \\
\hline ILC:SC & 0.73 & 0.57 & 0.89 & 0.02 & & & & \\
\hline MCB:SC & 1.25 & 0.90 & 1.60 & 0.05 & & & & \\
\hline Molecular_subtyping & & & & & & & & \\
\hline Luminal:TNBC & 0.79 & 0.46 & 1.12 & 0.02 & & & & \\
\hline HER2(+):TNBC & 0.58 & 0.29 & 0.87 & 0.03 & & & & \\
\hline LH:TNBC & 0.24 & 0.11 & 0.59 & 0.01 & & & & \\
\hline NLR & & & & & & & & \\
\hline$\geq 3.14:<3.14$ & 1.09 & 0.60 & 1.58 & $<0.01$ & & & & \\
\hline Model4 & & & & & 0.05 & 0.07 & 0.775 & 332.20 \\
\hline Tumor_size*, cm & 0.88 & 0.47 & 1.29 & 0.02 & & & & \\
\hline Age*, year & 1.07 & 0.66 & 1.48 & 0.05 & & & & \\
\hline Ki67 & & & & & & & & \\
\hline$\geq 0.50:<0.50$ & 1.22 & 0.75 & 1.69 & 0.03 & & & & \\
\hline Pathology & & & & & & & & \\
\hline IDC:SC & 0.46 & 0.30 & 0.62 & $<0.01$ & & & & \\
\hline ILC:SC & 0.45 & 0.31 & 0.59 & $<0.01$ & & & & \\
\hline MCB:SC & 1.21 & 0.79 & 1.62 & $<0.01$ & & & & \\
\hline Molecular_subtyping & & & & & & & & \\
\hline Luminal:TNBC & 0.85 & 0.48 & 1.22 & 0.02 & & & & \\
\hline HER2(+):TNBC & 0.57 & 0.02 & 1.12 & $<0.01$ & & & & \\
\hline LH:TNBC & 0.77 & 0.36 & 1.18 & $<0.01$ & & & & \\
\hline Blood_flow & & & & & & & & \\
\hline$\geq 1.88:<1.88$ & 1.25 & 0.84 & 1.66 & 0.01 & & & & \\
\hline NLR & & & & & & & & \\
\hline$\geq 3.14:<3.14$ & 1.17 & 0.82 & 1.52 & $<0.01$ & & & & \\
\hline BMI & & & & & & & & \\
\hline$<18: 18-24$ & 0.91 & 0.53 & 1.28 & $<0.01$ & & & & \\
\hline$\geq 25: 18-24$ & 1.03 & 0.64 & 1.42 & $<0.01$ & & & & \\
\hline
\end{tabular}


Table I (Continued).

\begin{tabular}{|c|c|c|c|c|c|c|c|c|}
\hline \multirow[t]{2}{*}{ Model } & \multicolumn{4}{|c|}{$95 \% \mathrm{Cl}$} & \multicolumn{4}{|c|}{ Discrimination } \\
\hline & OR & Lower & Upper & P-value & Brier & $\mathbf{R}^{2}$ & C-Index & AIC \\
\hline Model5 & & & & & 0.04 & 0.05 & 0.796 & 334.50 \\
\hline Tumor_size*, cm & 1.12 & 0.77 & 1.47 & 0.05 & & & & \\
\hline $\begin{array}{l}\text { Pathology } \\
\text { IDC:SC } \\
\text { ILC:SC } \\
\text { MCB:SC }\end{array}$ & $\begin{array}{l}0.67 \\
0.58 \\
1.42\end{array}$ & $\begin{array}{l}0.32 \\
0.40 \\
1.05\end{array}$ & $\begin{array}{l}1.02 \\
0.76 \\
1.77\end{array}$ & $\begin{array}{c}0.04 \\
0.02 \\
<0.01\end{array}$ & & & & \\
\hline $\begin{array}{l}\text { Molecular_subtyping } \\
\text { Luminal:TNBC } \\
\text { HER2(+):TNBC } \\
\text { LH:TNBC }\end{array}$ & $\begin{array}{l}0.78 \\
0.68 \\
0.67\end{array}$ & $\begin{array}{l}0.37 \\
0.31 \\
0.32\end{array}$ & $\begin{array}{l}1.19 \\
1.05 \\
1.02\end{array}$ & $\begin{array}{l}<0.01 \\
<0.01 \\
<0.01\end{array}$ & & & & \\
\hline $\begin{array}{l}K i 67 \\
\geq 0.50:<0.50\end{array}$ & 1.24 & 0.81 & 1.67 & 0.02 & & & & \\
\hline $\begin{array}{l}\text { NLR } \\
\geq 3.14:<3.14\end{array}$ & 1.13 & 0.80 & 1.46 & 0.04 & & & & \\
\hline $\begin{array}{l}\text { BMI } \\
<18: 18-24 \\
\geq 25: 18-24\end{array}$ & $\begin{array}{l}0.72 \\
0.98\end{array}$ & $\begin{array}{l}0.41 \\
0.49\end{array}$ & $\begin{array}{l}1.03 \\
1.47\end{array}$ & $\begin{array}{l}<0.01 \\
<0.01\end{array}$ & & & & \\
\hline
\end{tabular}

Note: *Continuous variable. Clinicopathological characteristics of 225 breast cancer patients treated with neoadjuvant chemotherapy.

Abbreviations: AIC, Akaike Information Criterion; IDC, invasive ductal carcinoma; ILC, invasive lobular carcinoma; SC, simplex carcinoma; MCB, medullary carcinoma of breast; LH, luminal-HER2(+); TNBC, triple negative breast cancer; BMI, body mass index; PI, pulsatility index; RI, resistance index; NLR, neutrophil lymphocyte ratio; OR, odds ratio; $95 \% \mathrm{Cl}, 95 \%$ confidence interval.

response in breast cancer patients who received NACT. Multivariate logistic analysis based on AIC and candidate variables are summarized in Table 1.

\section{Development and Validation of}

\section{a Predictive Nomogram for Pathological \\ Response}

Next, we optimized the model by performing a stepwise regression analysis. Multivariate analysis showed that tumor size $(0.83 ; 95 \% \mathrm{CI}, 0.72$ to 0.97$)$, PI ( $1.05,95 \%$ CI, 0.87 to 1.27$)$, RI $(1.19,95 \%$ CI, 0.93 to 1.51$)$, pathology (IDC:SC, $0.30 ; 95 \% \mathrm{CI}, 0.14$ to 0.63 ; ILC: $\mathrm{SC}, 0.48 ; 95 \% \mathrm{CI}, 0.35$ to 0.66 ; MCB:SC, $1.52 ; 95 \%$ CI, 0.93 to 2.49), molecular subtyping (Luminal:TNBC, 0.95 ; $95 \%$ CI, 0.74 to 1.21 ; HER2(+):TNBC, $0.58 ; 95 \%$ CI, 0.45 to 0.75 ; LH:TNBC, $0.74 ; 95 \%$ CI, 0.55 to $0.99)$, blood flow $(1.34 ; 95 \% \mathrm{CI}, 1.02$ to 1.78$)$, NLR (1.18; 95\% CI, 0.67 to 2.09$)$, BMI $(<18: 18-24,0.98$; $95 \%$ CI, 0.72 to 1.35 ; $\geq 25: 18-24,1.53 ; 95 \%$ CI, 1.18 to 1.98) were independently associated with pathological response.

\section{Assessment and Validation of the Models}

The performance of the nomogram score exhibited a robust C-index of 0.89 ( $95 \%$ confidence interval [CI]: 0.83 to 0.95 ) in the training cohort and a high C-index of 0.87 ( $95 \%$ CI: 0.81 to 0.93 ) in the validation cohort. Calibration curves clearly showed that the nomogram produced robust results in the training cohort and testing cohort, respectively (Figure 3). Besides, incorporating ultrasound imaging and clinical indices achieved an AUC of 0.89 (95\% CI: 0.9464-0.9778) in the identification of pathological response with an accuracy of 0.89 (95\% CI: 90.1-94.4\%) depending on individual clinical indicators or with an accuracy of 0.89 (95\% CI: 90.1-94.4\%) via ultrasound imaging index (Figure 4A). To further test the efficacy of the model, we used clinical impact curves to evaluate stratified risk. The calibration curve showed that the risk of pathological response could be readily distinguished in patients with breast cancer (Figure 4B).

\section{Discussion}

Many studies to date have evaluated the predictive ability of markers as a means of assessing breast cancer patients 
Table 2 The Predictive Ability and Parameter Inclusion of Prediction Models Reported in Previous Literature

\begin{tabular}{|c|c|c|c|c|}
\hline \multirow[t]{2}{*}{ Variables } & \multirow[b]{2}{*}{ OR } & \multicolumn{2}{|l|}{$95 \% \mathrm{Cl}$} & \multirow[t]{2}{*}{ P-value } \\
\hline & & Lower & Upper & \\
\hline Tumor_size*, cm & 0.83 & 0.72 & 0.97 & $<0.01$ \\
\hline PI & & & & \\
\hline$<0.58: \geq 0.58$ & 1.05 & 0.87 & 1.27 & 0.02 \\
\hline $\mathbf{R I}$ & & & & \\
\hline$\geq 0.47:<0.47$ & 1.19 & 0.93 & 1.51 & 0.04 \\
\hline Pathology & & & & \\
\hline IDC:SC & 0.30 & 0.14 & 0.63 & 0.01 \\
\hline ILC:SC & 0.48 & 0.35 & 0.66 & 0.02 \\
\hline MCB:SC & 1.52 & 0.93 & 2.49 & $<0.01$ \\
\hline Molecular_subtyping & & & & \\
\hline Luminal:TNBC & 0.95 & 0.74 & 1.21 & $<0.01$ \\
\hline HER2(+):TNBC & 0.58 & 0.45 & 0.75 & 0.03 \\
\hline LH:TNBC & 0.74 & 0.55 & 0.99 & 0.03 \\
\hline Blood_flow & & & & \\
\hline$\geq 1.88:<1.88$ & 1.34 & 1.02 & 1.78 & 0.04 \\
\hline NLR & & & & \\
\hline$\geq 3.14:<3.14$ & 1.18 & 0.67 & 2.09 & $<0.01$ \\
\hline BMI & & & & \\
\hline$<18: 18-24$ & 0.98 & 0.72 & 1.35 & $<0.01$ \\
\hline$\geq 25: 18-24$ & 1.53 & 1.18 & 1.98 & 0.02 \\
\hline
\end{tabular}

Note: *Continuous variable. Multivariate logistic regression analysis for risk factors associated with rNACT in patients with breast cancer.

Abbreviations: IDC, invasive ductal carcinoma; ILC, invasive lobular carcinoma; SC, simplex carcinoma; MCB, medullary carcinoma of breast; LH, luminal-HER2(+); TNBC, triple negative breast cancer; BMI, body mass index; PI, pulsatility index; RI, resistance index; NLR, neutrophil lymphocyte ratio.

who achieved NACT. ${ }^{26-28}$ In early breast cancer, NACT can achieve an ideal complete or partial clinical response, which can lead to a better frequency of breast conserving therapy than adjuvant chemotherapy. Indeed, NACT can make breast conserving surgery more feasible, and is more likely to eradicate micrometastasis than NACT given after operation. ${ }^{29}$ However, most patients did not achieve the desired expected results, and the reported clinical response rate was between $6 \%$ and $45 \% .^{30,31}$ In addition, the clinical response status is usually determined only by the surgical specimens after NACT. Herein, appropriate markers of chemosensitivity may help select patients who most benefit from NACT. As is shown in Table 2, studies have also demonstrated that clinicopathological parameters, particularly genetic and molecular phenotypes can be used to predict NACT treatment outcomes. However, the expensive cost, low efficiency of predicting outcomes, and non-universal detection methods are not conducive to the vigorous promotion of these prediction models. As such, identifying reliable predictive factors for NACT in breast cancer remains a challenge because these parameters are not simple, conventional, objective, and inexpensive laboratory indexes. Collectively, the high costs associated with new biomarkers limited their utility in regular clinical practice.

Our study also demonstrated several factors that were significantly associated with pathological response and could be used as reliable predictive factors, including tumor size, PI, RI, pathology, molecular subtyping, blood flow classification, and BMI. Thus, we developed and validated a novel nomogram model to predict the probability of pathological response to NACT; we named this pathological response to NACT score (pre-rNACT score). This predictive model is a visualized scale that is calculated by weighted variables, including molecular biomarkers, medical imaging tests, and histomorphological factors. If breast cancer patients required NACT, then the clinician could rely on this pre-rNACT score to assess the risk of pathological response; this may help the clinician to decide on further treatments during the follow-up period. To verify whether patients could benefit from pre-NACT scoring, we used receiver operating characteristic curves and clinical impact curves. Based on pretreatment clinical factors and simple laboratory indexes, we found that the optimal decision cutoff point was achieved when the risk of pathological response to the NACT threshold was set to 0.57 ; this represented a point at which there was significant discriminative power. To our knowledge, we successfully used ultrasound imaging indicators to integrate clinicopathological indicators to predict the efficacy of NACT, which demonstrated that a robust model could help breast cancer patients evaluate the risk of pathological response to NACT.

In our study, we found that NLR was highly associated with pathological response, which was consistent with the results of a prior study. ${ }^{32,33}$ Indeed, inflammation is a key driver of cancer development and progression and is closely linked to metastasis. ${ }^{34}$ Neutrophils acted as an important component of the tumor-induced inflammatory response, whereas lymphocytes are most often related to anti-tumor immune responses such that NLR values can reflect the dynamic balance between inflammatory and anti-inflammatory responses in cancer patients. ${ }^{21}$ Besides, the tumor immunoediting theory highlights the fact that different stages of the inflammatory response can 

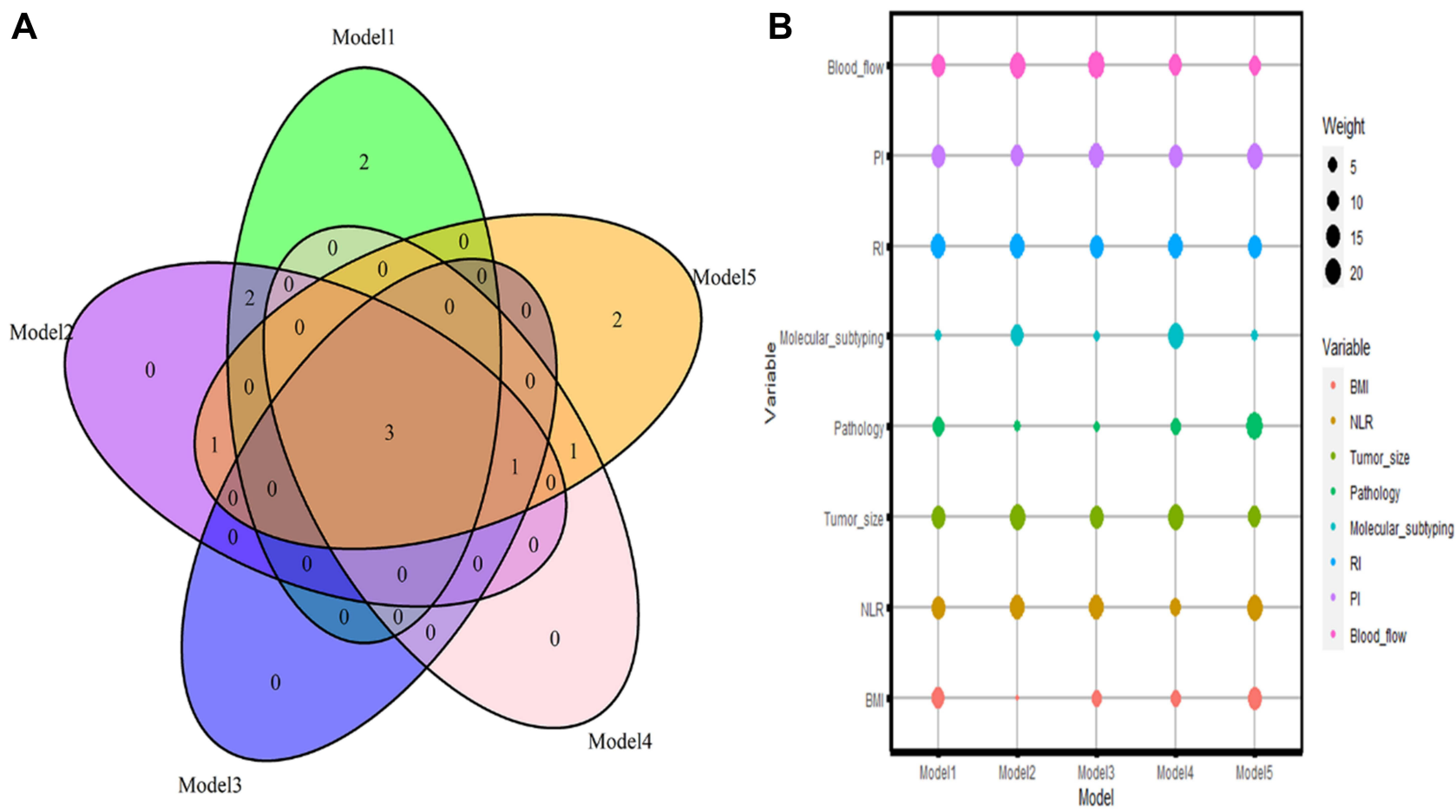

Figure 2 Statistical analysis of features included in models by Akaike Information Criterion. (A) Venn diagrams showing candidate variables for predicting the degree of pathological remission in five models. (B) Scaled importance rank of all features included in nomogram for identifying the possibility of pathological remission.

A Tumor_size
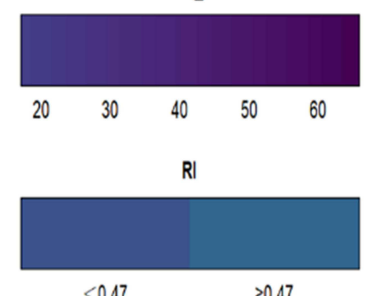

Molecular_subtyping
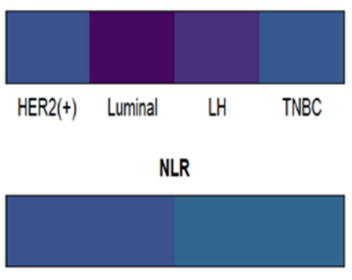

$<3.14$

$\geq 3.14$
PI
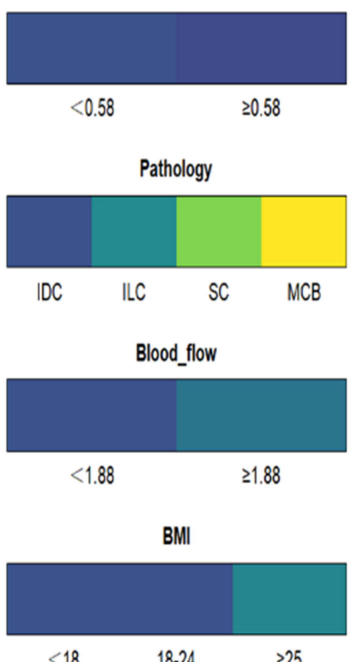

Score

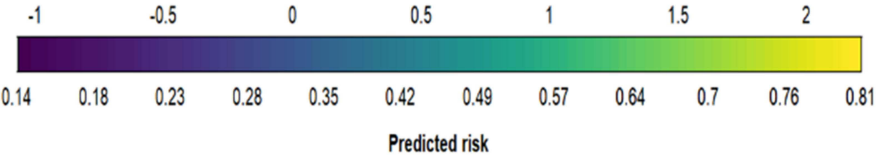

B

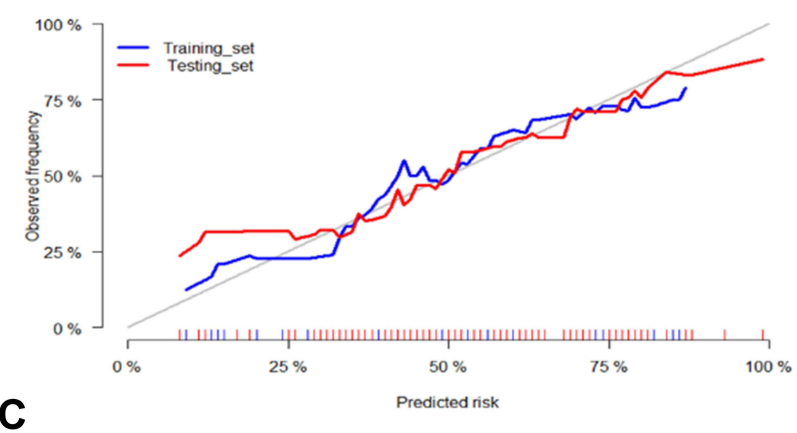

C

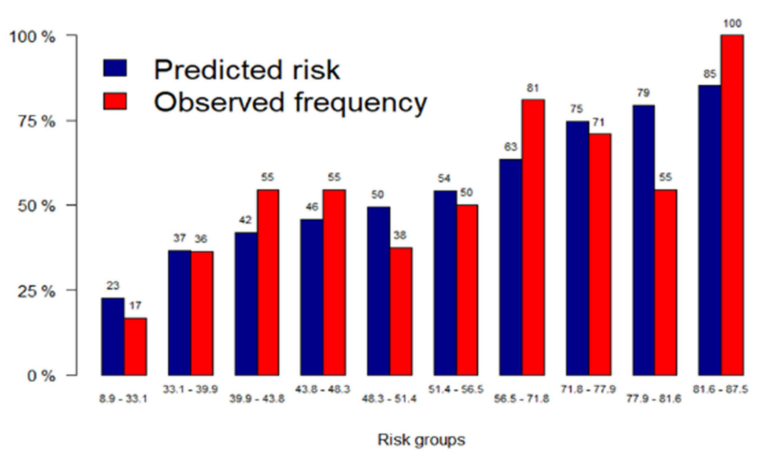

Figure 3 Generalized linear model. (A) Nomogram conveying the results of the candidate factors for predicting the possibility of pathological remission. (B) Calibration curves for internal validation of the nomogram (blue line represented training set, the red line represented testing set). (C) Predicted risk histogram comparing predicted risk of the nomogram with the observed frequency. 
A

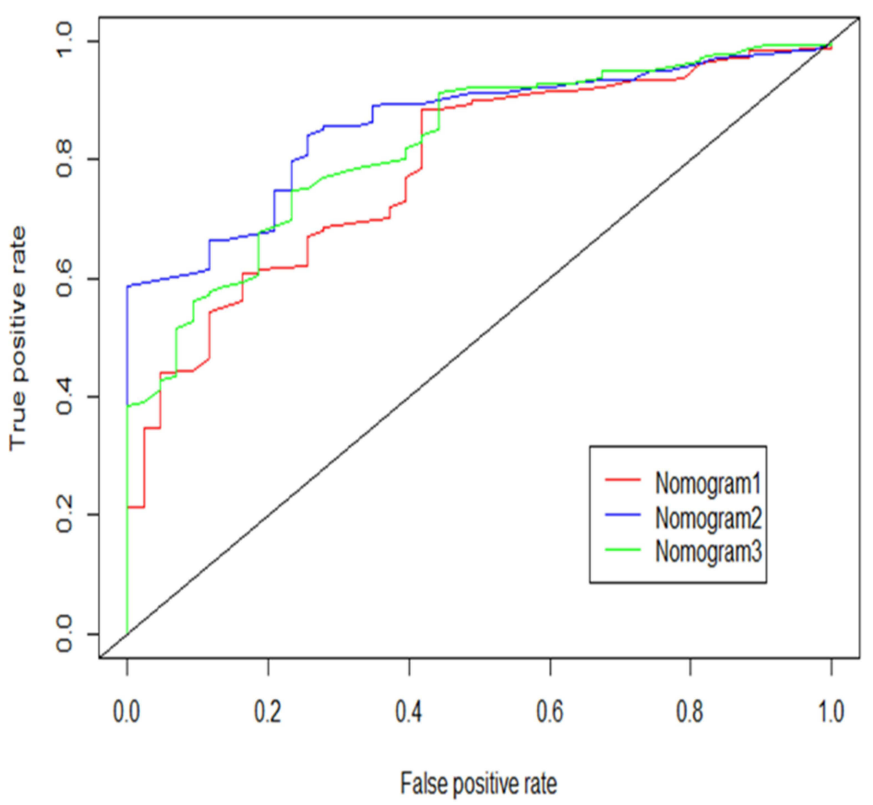

B

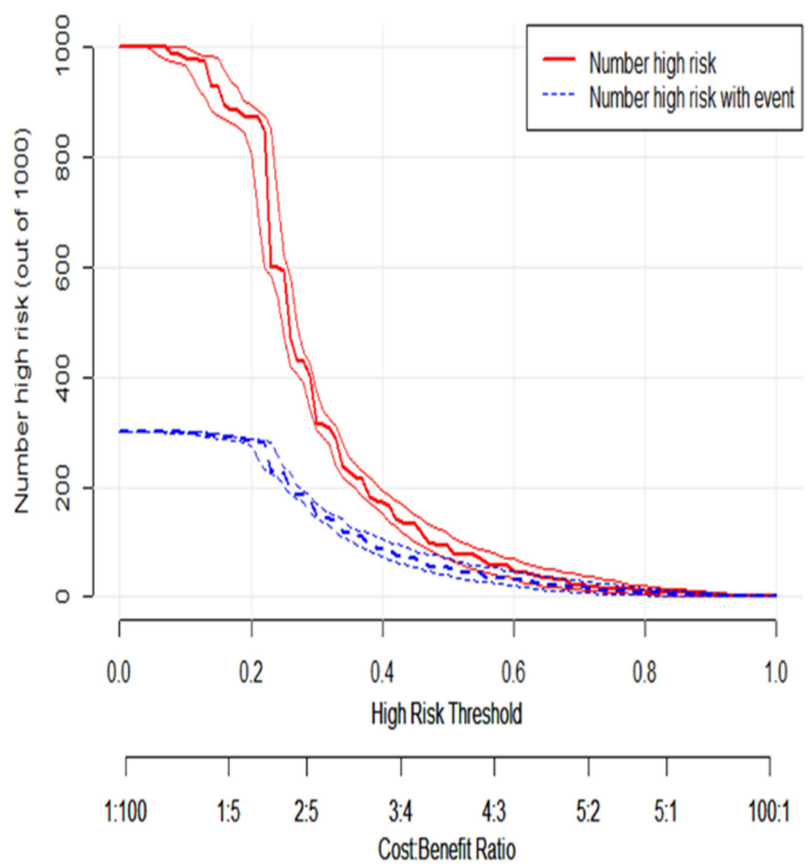

Figure 4 Predictive performance of nomogram based on ultrasound imaging and clinical indices. (A) AUC for predicting the possibility of pathological remission (Nomograml. Ultrasound imaging index; Nomogram2. Ultrasound imaging index, Clinicopathological parameters; Nomogram3. Clinicopathological parameters). (B) Clinical impact curve for the nomogram score (nomogram2).

contribute to the generation and development of tumors. ${ }^{35}$ In total, pretreatment NLR values served as a readout for inflammatory status, which has been employed to evaluate treatment efficacy and patient prognosis in cancer patients undergoing chemotherapy.

Previous studies have routinely used novel biomarkers to create nomograms. In this study, we sought to incorporate ultrasound image parameters, including PI, RI, and blood flow classification. Axillary ultrasound for restaging after NACT has been suggested to reduce the false-negative rates of sentinel lymph node biopsy. ${ }^{36}$ However, even with the combined model constructed in previous studies through integration of imaging and clinicopathologic characteristics, the sensitivity and specificity for predicting axillary pathological response to NACT are moderate, $67.9 \%$, and $73.6 \%$, respectively. ${ }^{13}$ In contrast, we created an accurate nomogram by combining the results of clinicopathologic factors, imaging indicators, and serological tumor indicators. These variables are the most common clinical indicators and are therefore very easy to acquire. Other indicators can therefore be avoided, thus reducing the financial burden incurred by patients.

Nevertheless, the present study has several limitations that need to be considered. First, our observations were limited to retrospective studies from a single center, and it is now necessary to conduct external validation using data from other centers. Second, the sample size was relatively small, and the predictive ability of the model needs to be further verified in large-sample studies. Third, because the model parameters were based on clinical peripheral blood and imaging results, the application of specific technologies (such as immunological diagnosis biomarkers) might improve the accuracy of our nomogram. Future research should be cautious and validated carefully.

\section{Conclusion}

We developed the pre-rNACT score scale, a novel nomogram-based prediction model featuring clinicopathologic factors and imaging results that can provide an optimized preoperative estimation of the risk of pathological response to NACT in patients with breast cancer.

\section{Acknowledgments}

The authors gratefully acknowledge all of our participants for sharing their medical records. The authors also wish to thank the staff members at Sixth Hospital of Ning Bo City of Zhe Jiang Province for their assistance with data collection.

\section{Funding}

There is no funding to report. 


\section{Disclosure}

None of the authors have any conflicts of interest to declare.

\section{References}

1. DeSantis CE, Ma J, Gaudet MM, et al. Breast cancer statistics, 2019. CA Cancer J Clin. 2019;69(6):438-451.

2. Vugts G, Maaskant-Braat AJ, Nieuwenhuijzen GA, Roumen RM, Luiten EJ, Voogd AC. Patterns of care in the administration of neo-adjuvant chemotherapy for breast cancer. a population-based study. Breast J. 2016;22(3):316-321. doi:10.1111/tbj.12568

3. Clough KB, Acosta-Marín V, Nos C, et al. Rates of neoadjuvant chemotherapy and oncoplastic surgery for breast cancer surgery: a French National Survey. Ann Surg Oncol. 2015;22 (11):3504-3511. doi:10.1245/s10434-015-4378-6

4. Cortazar P, Zhang L, Untch M, et al. Pathological complete response and long-term clinical benefit in breast cancer: the CTNeoBC pooled analysis. Lancet. 2014;384(9938):164-172. doi:10.1016/S01406736(13)62422-8

5. Abrial SC, Penault-Llorca F, Delva R, et al. High prognostic significance of residual disease after neoadjuvant chemotherapy: a retrospective study in 710 patients with operable breast cancer. Breast Cancer Res Treat. 2005;94(3):255-263. doi:10.1007/s10549005-9008-8

6. Rouzier R, Extra JM, Klijanienko J, et al. Incidence and prognostic significance of complete axillary downstaging after primary chemotherapy in breast cancer patients with $\mathrm{T} 1$ to $\mathrm{T} 3$ tumors and cytologically proven axillary metastatic lymph nodes. J Clin Oncol. 2002;20(5):1304-1310. doi:10.1200/JCO.2002.20.5.1304

7. Caudle AS, Yang WT, Mittendorf EA, et al. Selective surgical localization of axillary lymph nodes containing metastases in patients with breast cancer: a prospective feasibility trial. JAMA Surg. 2015;150(2):137-143. doi:10.1001/jamasurg.2014.1086

8. Hennessy BT, Hortobagyi GN, Rouzier R, et al. Outcome after pathologic complete eradication of cytologically proven breast cancer axillary node metastases following primary chemotherapy. $J$ Clin Oncol. 2005;23(36):9304-9311. doi:10.1200/JCO.2005.02.5023

9. Hurvitz SA, Martin M, Symmans WF, et al. Neoadjuvant trastuzumab, pertuzumab, and chemotherapy versus trastuzumab emtansine plus pertuzumab in patients with HER2-positive breast cancer (KRISTINE): a randomised, open-label, multicentre, Phase 3 trial. Lancet Oncol. 2018;19(1):115-126. doi:10.1016/S1470-2045(17) 30716-7

10. Kantor O, Sipsy LM, Yao K, James TA, Predictive A. Model for axillary node pathologic complete response after neoadjuvant chemotherapy for breast cancer. Ann Surg Oncol. 2018;25(5):1304-1311. doi:10.1245/s10434-018-6345-5

11. Davis J Jr, Hoskin TL, Day CN, et al. Performance and clinical utility of models predicting eradication of nodal disease in patients with clinically node-positive breast cancer treated with neoadjuvant chemotherapy by tumor biology. Ann Surg Oncol. 2020;27 (12):4678-4686. doi:10.1245/s10434-020-08885-w

12. Zhang J, Xiao L, Pu S, Liu Y, He J, Wang K. Can we reliably identify the pathological outcomes of neoadjuvant chemotherapy in patients with breast cancer? Development and validation of a logistic regression nomogram based on preoperative factors. Ann Surg Oncol. 2021;28(5):2632-2645. doi:10.1245/s10434-020-09214-x

13. Kim WH, Kim HJ, Park HY, et al. Axillary pathologic complete response to neoadjuvant chemotherapy in clinically node-positive breast cancer patients: a predictive model integrating the imaging characteristics of ultrasound restaging with known clinicopathologic characteristics. Ultrasound Med Biol. 2019;45(3):702-709. doi:10.1016/j.ultrasmedbio.2018.10.026
14. Hwang HW, Jung H, Hyeon J, et al. A nomogram to predict pathologic complete response (pCR) and the value of tumor-infiltrating lymphocytes (TILs) for prediction of response to neoadjuvant chemotherapy (NAC) in breast cancer patients. Breast Cancer Res Treat. 2019;173(2):255-266. doi:10.1007/s10549-018-4981-x

15. Matsuda N, Hayashi N, Ohde S, et al. A nomogram for predicting locoregional recurrence in primary breast cancer patients who received breast-conserving surgery after neoadjuvant chemotherapy. J Surg Oncol. 2014;109(8):764-769. doi:10.1002/jso.23586

16. Park KU, Chen Y, Chitale D, et al. Utilization of the 21-gene recurrence score in a diverse breast cancer patient population: development of a clinicopathologic model to predict high-risk scores and response to neoadjuvant chemotherapy. Ann Surg Oncol. 2018;25 (7):1921-1927. doi:10.1245/s10434-018-6440-7

17. Tabchy A, Valero V, Vidaurre $T$, et al. Evaluation of a 30-gene paclitaxel, fluorouracil, doxorubicin, and cyclophosphamide chemotherapy response predictor in a multicenter randomized trial in breast cancer. Clin Cancer Res. 2010;16(21):5351-5361. doi:10.1158/1078-0432.CCR-10-1265

18. Vila J, Mittendorf EA, Farante G, et al. Nomograms for predicting axillary response to neoadjuvant chemotherapy in clinically node-positive patients with breast cancer. Ann Surg Oncol. 2016;23 (11):3501-3509. doi:10.1245/s10434-016-5277-1

19. Zhang F, Huang M, Zhou H, et al. A nomogram to predict the pathologic complete response of neoadjuvant chemotherapy in triple-negative breast cancer based on simple laboratory indicators. Ann Surg Oncol. 2019;26(12):3912-3919. doi:10.1245/s10434-01907655-7

20. Keam B, Im SA, Park S, et al. Nomogram predicting clinical outcomes in breast cancer patients treated with neoadjuvant chemotherapy. J Cancer Res Clin Oncol. 2011;137(9):1301-1308. doi:10.1007/s00432-011-0991-3

21. Zhu J, Jiao D, Zhao Y, et al. Development of a predictive model utilizing the neutrophil to lymphocyte ratio to predict neoadjuvant chemotherapy efficacy in early breast cancer patients. Sci Rep. 2021;11(1):1350. doi:10.1038/s41598-020-80037-2

22. Pu S, Wang K, Liu Y, et al. Nomogram-derived prediction of pathologic complete response (pCR) in breast cancer patients treated with neoadjuvant chemotherapy (NCT). BMC Cancer. 2020;20(1):1120. doi:10.1186/s12885-020-07621-7

23. Kim SY, Cho N, Choi Y, et al. Factors affecting pathologic complete response following neoadjuvant chemotherapy in breast cancer: development and validation of a predictive nomogram. Radiology. 2021;299(2):290-300. doi:10.1148/radiol.2021203871

24. Eisenhauer EA, Therasse P, Bogaerts J, et al. New response evaluation criteria in solid tumours: revised RECIST guideline (version 1.1). Eur J Cancer. 2009;45(2):228-247. doi:10.1016/j.ejca.2008.10.026

25. Dziak JJ, Coffman DL, Lanza ST, Li R, Jermiin LS. Sensitivity and specificity of information criteria. Brief Bioinform. 2020;21 (2):553-565. doi:10.1093/bib/bbz016

26. Corbeau I, Jacot W, Guiu S. Neutrophil to lymphocyte ratio as prognostic and predictive factor in breast cancer patients: a systematic review. Cancers. 2020;12(4). doi:10.3390/cancers 12040958

27. Okuturlar Y, Gunaldi M, Tiken EE, et al. Utility of peripheral blood parameters in predicting breast cancer risk. Asian Pac J Cancer Prev. 2015;16(6):2409-2412. doi:10.7314/APJCP.2015.16.6.2409

28. Graziano V, Grassadonia A, Iezzi L, et al. Combination of peripheral neutrophil-to-lymphocyte ratio and platelet-to-lymphocyte ratio is predictive of pathological complete response after neoadjuvant chemotherapy in breast cancer patients. Breast. 2019;44:33-38. doi:10.1016/j.breast.2018.12.014

29. Asselain B, Barlow W, Bartlett J. Long-term outcomes for neoadjuvant versus adjuvant chemotherapy in early breast cancer: meta-analysis of individual patient data from ten randomised trials. Lancet Oncol. 2018;19(1):27-39. doi:10.1016/S1470-2045(17) $30777-5$ 
30. von Minckwitz G, Untch M, Blohmer JU, et al. Definition and impact of pathologic complete response on prognosis after neoadjuvan chemotherapy in various intrinsic breast cancer subtypes. J Clin Oncol. 2012;30(15):1796-1804. doi:10.1200/JCO.2011.38.8595

31. Kong X, Moran MS, Zhang N, Haffty B, Yang Q. Meta-analysis confirms achieving pathological complete response after neoadjuvant chemotherapy predicts favourable prognosis for breast cancer patients. Eur J Cancer. 2011;47(14):2084-2090. doi:10.1016/j.ejca.2011.06.014

32. Asano Y, Kashiwagi S, Onoda N, et al. Predictive value of neutrophil/ lymphocyte ratio for efficacy of preoperative chemotherapy in triple-negative breast cancer. Ann Surg Oncol. 2016;23 (4):1104-1110. doi:10.1245/s10434-015-4934-0

33. Bae SJ, Cha YJ, Yoon C, et al. Prognostic value of neutrophil-tolymphocyte ratio in human epidermal growth factor receptor 2-negative breast cancer patients who received neoadjuvant chemotherapy. Sci Rep. 2020;10(1):13078. doi:10.1038/s41598-020-69965-1
34. Elinav E, Nowarski R, Thaiss CA, Hu B, Jin C, Flavell RA. Inflammation-induced cancer: crosstalk between tumours, immune cells and microorganisms. Nat Rev Cancer. 2013;13(11):759-771. doi: $10.1038 / \mathrm{nrc} 3611$

35. Bui JD, Schreiber RD. Cancer immunosurveillance, immunoediting and inflammation: independent or interdependent processes? Curr Opin Immunol. 2007;19(2):203-208. doi:10.1016/j.coi.2007.02.001

36. Fu JF, Chen HL, Yang J, Yi CH, Zheng S. Feasibility and accuracy of sentinel lymph node biopsy in clinically node-positive breast cancer after neoadjuvant chemotherapy: a meta-analysis. PLoS One. 2014;9 (9):e105316. doi:10.1371/journal.pone.0105316

\section{Publish your work in this journal}

Cancer Management and Research is an international, peer-reviewed open access journal focusing on cancer research and the optimal use of preventative and integrated treatment interventions to achieve improved outcomes, enhanced survival and quality of life for the cancer patient.
The manuscript management system is completely online and includes a very quick and fair peer-review system, which is all easy to use. Visit http://www.dovepress.com/testimonials.php to read real quotes from published authors. 